\title{
Analysis of Optical Radiation Generated by Electrical Discharges on Support Insulator
}

\author{
P. FrącZ*, T. Boczar, D. ZMARZey AND T. SzcZYrba \\ Faculty of Electrical Engineering, Automatic Control and Computer Science, Opole University of Technology \\ Prószkowska 76, 45-758 Opole, Poland
}

\begin{abstract}
Subject of the paper concerns determination of the feasibility and indication of the application scope of optical spectroscopy methods in the diagnosis of high-voltage insulators. Results of measurements of optical radiation emitted by partial and complete electrical discharges occurring on a support insulator model placed in air are presented in the paper. Registration was performed using a spectrophotometer, enabling for measurement of optical radiation in the range from 200 to $1800 \mathrm{~nm}$. The primary objective of the laboratory tests was to evaluate the impact of supply voltage changes in the range from 0 to $60 \mathrm{kV}$ on the intensity and spectral character of the optical radiation emitted by electric discharges generated on the porcelain insulation surface of the support insulator. In the scope of studies analyzes of the effects of voltage changes on the obtained spectral wave forms were performed, whereby voltage increase and reduction was examined separately. Additionally, measurements and analyzes carried out included impact estimation of the time period as the voltage was applied to the high-voltage electrodes on the course of optical phenomena related to the generation of electrical discharges.
\end{abstract}

DOI: $10.12693 /$ APhysPolA.124.413

PACS: $79.60 . \mathrm{Bm}, 42.79 . \mathrm{Pw}, 52.80 . \mathrm{Hc}$

\section{Introduction}

One of the basic problems of insulation systems used in electrical equipment and high voltage transmission lines is the process of ageing, which progresses during their service and the deterioration of insulating properties caused by this process. Reduction in aging resistance is caused by a number of external factors, among which atmospheric, environmental and voltage exposures can be mentioned. Important aging factors that occur in service practice include: UV radiation, the presence of ozone and oxides of nitrogen, temperature fluctuations, rain (including acid rain), depositions of rime or dirt, and partial discharges (PDs). Relatively dangerous and not so deeply examined aging risk for insulators (especially composites) is rainfall, which may cause loss of hydrophobicity in insulator, and thus lead to increase in leakage of currents and to development of surface discharges [1].

The main objective of the diagnostic research is answering, as accurately as possible, the questions on the manner, time and duration of the insulation element deterioration process, and additionally on conditions under which the total damage will occur.

Diagnostic techniques are usually used to monitor selected parameters, criterion indices of which inform about the possibility of failure and permanent damage of the examined unit. In recent years, rapid development of various monitoring, analysis and estimation techniques for assessment of the operation state of electrical equipment,

*corresponding author; e-mail: pfracz@gmail.com being now an integral element of the energy power production, transmission and distribution processes [1-5].

Subject matter of this article is to determine feasibility and indicate the scope of the optical spectrophotometry methods for partial discharge (PD) detection, which can be generated on the surface of insulators. Stand-off insulators are designed for solid supporting of the conductive part, which has to be insulated from the ground or from another part under the voltage [4, 6-11]. They support current circuits or metallic caps of insulators connected with it, thus forming insulation columns. In addition, they are used to mount fuses and contacts in the disconnectors and switches. Post insulators are not exposed to significant mechanical stress during normal operation. However, they must withstand specific dynamic impacts caused by the flow of short-circuit currents [12-14]. Stand-off insulator is a classic and one of the simplest examples of the parallel stratified system, wherein the intensity of electric field on both sides of the thresholds in each of the dielectrics has the same value. With equal electric field strengths, electrical spark jump occurs at dielectric with the lowest strength. Equivalent circuits of this type of systems, in particular for the long rod insulators and pin insulator chain, equations describing physics of the analysed phenomena and graphs indicating dependences between the voltage spark and the distance between electrodes were widely discussed, among others, in $[1,9]$.

\section{Characteristics of test insulator and measurement system}

For the purpose of PD generation on the surface of stand-off insulator, a model system was developed, of 

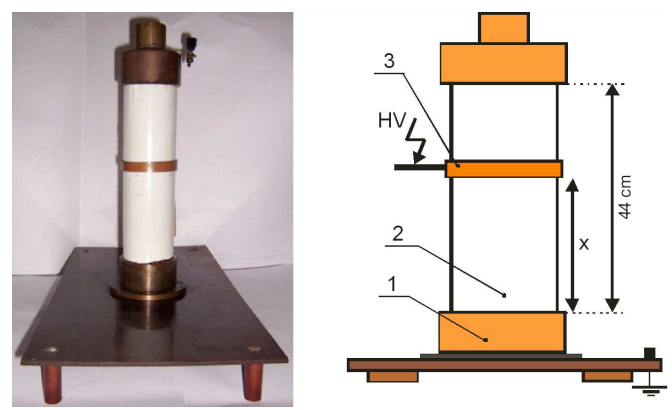

Fig. 1. Stand-off insulator modeling system — real view and schematic diagram, where: 1 - grounded fitting, 2 - ceramic insulation, 3 - movable metal clamp.

which schematic representation and the real view are presented in Fig. 1.

The examined post insulator model was powered by circuit that allows stepless regulation of the applied voltage in the range from 0 to $60 \mathrm{kV}$. Power system used consisted of an automatic control and voltage measurement circuit, a control panel and a testing transformer.

To measure optical radiation emitted by PDs generated in the insulator modeling system, a spectrophotometer was used with optical transducer placed in a holder made for this purpose, attached to a tripod. The tripod, which was used allows for adjustments of height, tilt angle, and distance between the measuring header and examined insulator. During the study, AVS-USB2000 spectrometer by OceanOptics, Inc. was used, the main element of which is a multi-stage diffraction grating that allows the analysis of optical spectra in the range of $270-1700 \mathrm{~nm}$ with resolution of about $0.5 \mathrm{~nm}$. Recorded signal is distributed to the grid through the fiber. After dispersion, the radiation falls on the CCD (Sony ILX511). The integration time can be adjusted in the range from $3 \mathrm{~ms}$ to $65 \mathrm{~s}$. Measurement in a particular element of the CCD (2048 elements of size $12.5 \times 200 \mu \mathrm{m}^{2}$ ) consists in counting of photons per unit time. One count corresponds to activation by 86 photons, which is equivalent to sensitivity of $2.9 \times 10^{-17} \mathrm{~J} /$ impulse. Relative sensitivity depends on the length of the analysed wave. The RMS value of the dark current is in the range from 2.5 to 4 counts. The dynamic range is 1300:1, and linearity is equal to $99.8 \%$. Detailed characteristics of spectrophotometer are presented in $[1,9]$. The device was connected with a $\mathrm{PC}$ through a serial port. For triggering measurements and initial preview of the obtained spectral dependences, standard software offered by OceanOptics, Inc. was used, whereas, for achieving purposes and further processing of the recorded measurement data, numerical procedures implemented in the Matlab programming environment were used.

\section{Test result analysis}

The scope of measurements performed on optical radiation emitted by PD generated on stand-off insulator modelling system covered following cases:

(a) Impact assessment of supply voltage value changes in the range from 0 to $0.99 U_{\mathrm{p}}$ (breakdown voltage) on the intensity and nature of wave forms of optical radiation spectra, indicated for the range from 0 to $1700 \mathrm{~nm}$. Moreover, under the research tests was performed a comparative analysis of the calculated spectra, obtained by lowering and raising the supply voltage.

(b) Estimation of the effect of PD generation time in the range from 0 to $25 \mathrm{~min}$ to the obtained wave forms of the emitted optical radiation spectra. Measurements were made with the same supply voltage equal to $0.99 U_{\mathrm{p}}$.

In order to perform comparative analyses of the obtained results, research tests, the scope of which were defined in points (a) and (b), were conducted at the same distance $x=12 \mathrm{~cm}$ between the aluminium clip (label-3 in Fig. 1) to which high potential power supply and grounded ferrule (label-1 in Fig. 1) were connected. During each series of measurements, the distance between the spectrophotometer measurement head and the place of PD generation was constant and equal to $3 \mathrm{~cm}$. Measurements were performed in a darkened room of the laboratory for electric power transformer insulation at the Institute of Electric Power Engineering and Renewable Energy of the Technical University of Opole, without access to any additional light of radiation sources.

In Fig. 2 there are presented spectra of optical radiation emitted by the PD generated on a stand-off insulator, obtained at the increase (a) and decrease (b) supply voltage, respectively.

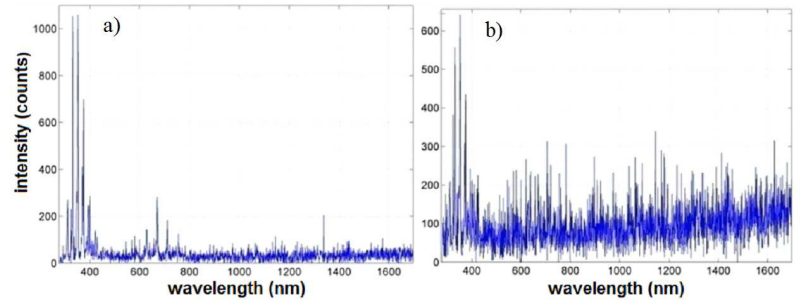

Fig. 2. Spectra of optical radiation emitted by PDs with increased (a) and decreased (b) supply voltage, set for $U=0.99 U_{\mathrm{p}}$, for the insulator model.

In order to determine the effect of the supply voltage control method on the obtained results of the measurements of optical radiation emitted by PDs, characteristics were determined which are presented in Fig. 3. They represent dependences of the intensity on the value of voltage and are plotted separately, when increased or decreased. Charts were determined for specific wavelengths, for which the highest values of intensity were reached in the spectra within the whole analysed range.

In Fig. 4 there are presented graphs of changes in the intensity of the optical radiation, determined for comparative purposes, for increasing (a) and decreasing (b) voltage supply value of the stand-off insulator modelling system. In order to expose the obtained dependences, graphs were determined within the limited wavelength 


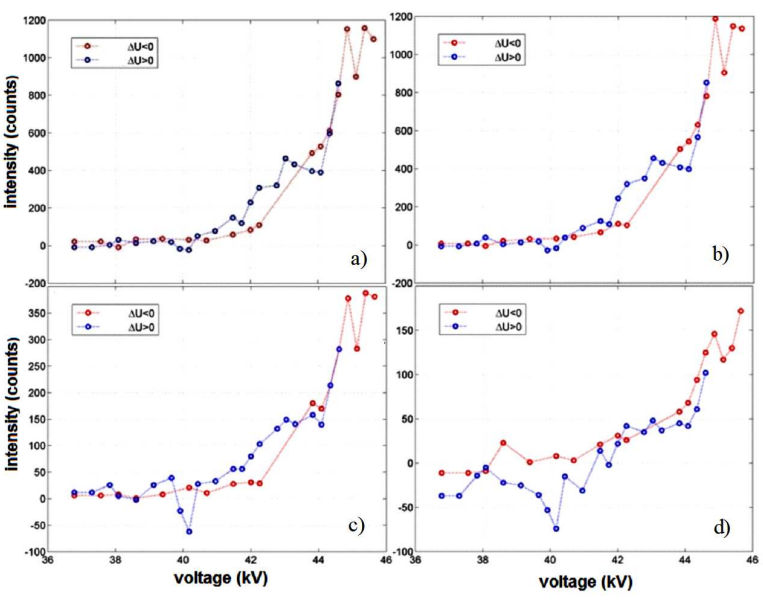

Fig. 3. Change of the intensity of optical radiation emitted by PDs at increase $(\Delta U>0)$ and decrease $(\Delta U<0)$ of the supply voltage for the selected wavelength $\lambda=333 \mathrm{~nm}(\mathrm{a}), \lambda=352 \mathrm{~nm}(\mathrm{~b}), \lambda=371 \mathrm{~nm}(\mathrm{c})$, $\lambda=711 \mathrm{~nm}(\mathrm{~d})$, for the stand-off insulator.

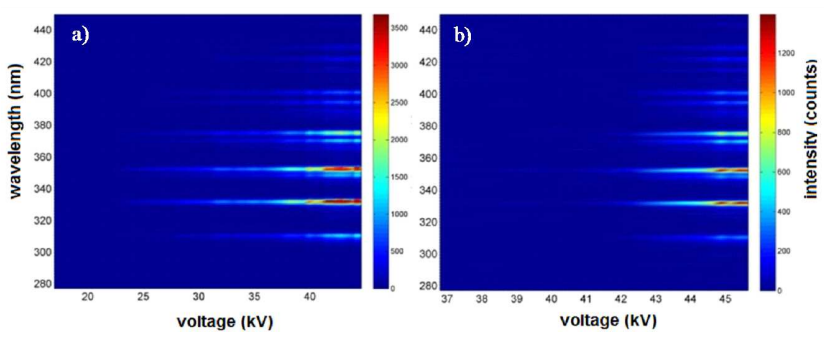

Fig. 4. Comparison of the intensity of optical radiation changes emitted by PDs at voltage rise (a) and drop (b), determined for wavelengths in the range $270-450 \mathrm{~nm}$, for the stand-off insulator.

range, that is $270-450 \mathrm{~nm}$, for which the highest value of intensity of the optical radiation was obtained.

In order to allow more accurate analysis of changes occurring for the obtained structures, a graph was developed that was restricted to maximum wavelengths in the spectrum, that is for the range from $270 \mathrm{~nm}$ to $450 \mathrm{~nm}$. Recording time was $25 \mathrm{~min}$. Intensity values are illustrated by a bar with palette of colours.

To determine the effect of PD generation duration on the shape of the calculated optical radiation spectra, a graph showing the dependence between the intensity and the emitted wavelength is presented in Fig. 5. Spectrum was determined for a time point of $15 \mathrm{~min}$, and was presented within the entire analysed wavelength range 0-1700 $\mathrm{nm}$ (a), and in the dominant wavelength range from $270 \mathrm{~nm}$ to $450 \mathrm{~nm}$.

\section{Summary}

Based on laboratory tests concerning registering optical radiation emitted by $\mathrm{PDs}$ generated on the model of

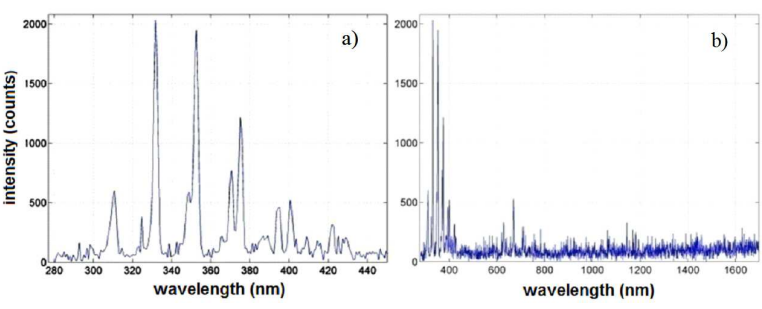

Fig. 5. Changes in intensity of optical radiation emitted by PDs as a function of time at constant supply voltage $U=0.99 U_{\mathrm{p}}$, for wavelength in the range $0-1700 \mathrm{~nm}$ and $270-450 \mathrm{~nm}$, time point of $15 \mathrm{~min}$, for stand-off insulation model.

stand-off insulator and on the analysis of the obtained results, the following conclusions can be formulated:

- Designated spectra have two local intensity extrema that exist in the ranges of approximately $280-430 \mathrm{~nm}$ and about 500-720 nm. Radiation intensity in the ultraviolet range, particularly in UV-A (this range was adopted based on ISO 21348:2007(E) Space environment natural and artificial - Process for determining solar irradiances), that means in the range between $315 \mathrm{~nm}$ and $400 \mathrm{~nm}$, is much higher than in the visible range $380-780 \mathrm{~nm}$. Radiation intensity values obtained in the $\mathrm{UV}-\mathrm{A}$ are on average more than three times higher than those registered in the visible range. For wavelengths exceeding $720 \mathrm{~nm}$, the calculated spectra have flat wave forms, without visible extremes. This indicates a relatively small infrared radiation (abbreviated IR) in the optical spectrum emitted by PDs. In particular this applies to near infrared (IR-A) radiation, that is in the range between $780 \mathrm{~nm}$ and $1400 \mathrm{~nm}$.

- In the designated optical radiation spectra are three dominant wavelengths: 333,352 , and $375 \mathrm{~nm}$, the intensity of which is decisively the largest in the whole analysed range. Intensity size corresponding to these wavelengths is differentiated according to the method of power supply control (decreasing, increasing). In addition to these maximum wavelengths in the ultraviolet range, several following lengths can be also distinguished: $312,389,394,401$, and $422 \mathrm{~nm}$, which are characterised by increased intensity values. They are independent of the power supply control method.

- In the visible range, three wavelengths can be identified, that is: 626,671 , and $711 \mathrm{~nm}$, for which the radiation intensity values are in this range the highest. It should be noted that values obtained for the three maximum wavelengths are on average more than three times lower than that in the UV-A range.

- Waveform pattern of the determined optical radiation spectra is independent of the voltage value of $\mathrm{PD}$ generation. This occurs in the voltage range from the ignition of discharges (approximately $0.5 U_{\mathrm{p}}$ ) to the value equal to $0.99 U_{\mathrm{p}}$. In minimum voltage values (less than $0.7 U_{\mathrm{p}}$ ), the intensity of radiation emitted by PDs is significantly lower and is characterized by low stabil- 
ity, and the examined phenomena are characterized by strong stochasticity threshold.

- The results showed no effect on the nature of the obtained dependences in changes of input voltage values, although it is increased or decreased. This concern both received wave forms of the optical radiation spectra, as well as the maximum intensity wavelength.

The nature of the calculated optical radiation spectra does not change over time (registration time $25 \mathrm{~min}$ ) for a given constant voltage value of $\mathrm{PD}$ generation. This proposal also applies to the maximum wavelength in the registered spectra. The observed changes concern absolute values of intensity size that is emitted by optical radiation for the distinguished wavelengths, which indicates fluctuating and largely stochastic nature of the generated PDs.

In summary, spectrophotometer application allowed registration and analysis of optical radiation within a wide spectral band, respectively in the range of $0 \mathrm{~nm}$ to $1700 \mathrm{~nm}$, which covered the ultraviolet, visible and infrared radiation range. The results indicate the largest energy contribution in the spectra of the registered electromagnetic waves emitted by the modeled PDs, UV-A radiation in the range $315-400 \mathrm{~nm}$. The optical spectroscopy method, applied in laboratory tests enables detection of partial discharges generated in the proposed stand-off insulator modeling system; however, this applies to supply voltages exceeding $0.7 U_{\mathrm{p}}$. Moreover, based on the performed spectral analysis, it was indicated that there are no dependences between the obtained results and the power supply control method (whether the power is increased or decreased), and the duration of partial discharge generation.

\section{Acknowledgments}

The work was financed with the means from the National Research and Development Center within LIDER program.

\section{References}

[1] P. Frącz, Use Optical Signals Emitted by Electrical Discharge in the Diagnosis Insulators, Opole University of Technology, Opole 2011 (in Polish).

[2] S. Borucki, T. Boczar, A. Cichoń, Europ. Phys. J. Spec. Top. 154, 23 (2008).

[3] T. Boczar, S. Borucki, A. Cichoń, M. Lorenc, Acta Phys. Pol. A 114, A21 (2008).

[4] S. Borucki, T. Boczar, A. Cichoń, Arch. Acoust. 36 49 (2011).

[5] S. Borucki, T. Boczar, A. Cichoń, Arch. Acoust. 32, Suppl., 291 (2007).

[6] M. Zdanowski, S. Wolny, D. Zmarzły, T. Boczar, J. Electrostat. 65, 239 (2007).

[7] D. Wotzka, T. Boczar, D. Zmarzły, Acta Phys. Pol. A 116, 428 (2009).

[8] T. Boczar, T. Malec, D. Wotzka, Acta Phys. Pol. A 122, 850 (2012).

[9] T. Boczar, D. Zmarzły, IEEE Trans. DEIS 13, 632 (2006).

[10] D. Wotzka, D. Zmarzły, T. Boczar, Acta Phys. Pol. A 118, 1272 (2010).

[11] T. Boczar, D. Zmarzły, Mater. Eval. 62, 935 (2004).

[12] T. Boczar, D. Zmarzły, J. Brit. Inst. Non-Destruct. Test. 45, 488 (2003).

[13] D. Wotzka, A. Cichoń, T. Boczar, Arch. Acoust. 37 19 (2012).

[14] D. Wotzka, T. Boczar, P. Frącz, Acta Phys. Pol. A 120, 767 (2011). 